\title{
КОМПЬЮТЕРНОЕ МОДЕЛИРОВАНИЕ УСТАНОВИВШИХСЯ РЕЖИМОВ РАБОТЫ ОДНОРОДНОЙ ЛИНИИ
}

\author{
3. И. Жолдыбаева, Е. Х. Зуслина, Б. Д. Утебалиева \\ НАО «Алматинский университет энергетики и связи», Алматьл, Республика Казахстан
}

DOI: https://doi.org/10.31435/rsglobal_ws/31072019/6585

\section{ARTICLE INFO}

Received: 19 May 2019

Accepted: 13 July 2019

Published: 31 July 2019

\section{KEYWORDS}

virtual laboratory works, congeneric lines, educational purposes. \begin{abstract}
Computer models of a congeneric loss and lossless line were developed on Delphi. These computer models are used to study the steady-state operating conditions of a congeneric line and allow to determine voltages, currents, power and resistance at any point and for different values of the primary parameters of the line, to build graphs of voltages and currents. Due to the fast speed of the program and the convenience of calculations, computer models can be used as virtual laboratory works for educational purposes in universities, colleges, or any scientific and technical institutions.
\end{abstract}

Citation: 3. И. Жолдыбаева, Е. Х. Зуслина, Б. Д. Утебалиева. (2019) Komp'yuternoe Modelirovanie Ustanovivshihsya Rezhimov Raboty Odnorodnoj Linii. World Science. 7(47), Vol.1. doi: 10.31435/rsglobal_ws/31072019/6585

Copyright: (C) 2019 3. И. Жолдыбаева, Е. Х. Зуслина, Б. Д. Утебалиева. This is an open-access article distributed under the terms of the Creative Commons Attribution License (CC BY). The use, distribution or reproduction in other forums is permitted, provided the original author(s) or licensor are credited and that the original publication in this journal is cited, in accordance with accepted academic practice. No use, distribution or reproduction is permitted which does not comply with these terms.

Введение. Одной из важных задач в теории электрических цепей с распределенными параметрами является изучение установившихся режимов работы однородный линии. Моделирование электромагнитных процессов в однородной линии с использованием физического эксперимента представляет значительные трудности. Применение компьютерного моделирования позволяет решить эту проблему. Широко применяемая программа моделирования Electronics Workbench позволяет моделировать однородные линии в виде четырехполюсников и определять напряжения, токи, мощности в начале и в конце линии $[1,2]$. Однако в процессе изучения дисциплины «Теория электрических цепей» раздела «Однородная линия» представляет большой интерес исследование распределения напряжения, тока, мощностей, сопротивления вдоль всей длины однородной линии для различных режимов её работы. С этой целью нами созданы программы для ЭВМ «Компьютерная модель длинной линии» [3] и «Исследование различных режимов в однородной линии без потерь с помощью компьютерного моделирования» [4]. При разработке данных компьютерных моделей учитывались основные задачи проектирования: разработка алгоритма и программирование, тестирование, отладка программ и их применение.

При проектировании компьютерных моделей однородной линии с потерями и без потерь была использована технология объектно-ориентированного программирования; написан пакет прикладных программ на языке Delphi в среде Embarcadero RAD Studio 2010. Дружественный интерфейс, большие возможности получения необходимых данных, скорость и стабильность работы - вот далеко не полный перечень достоинств компьютерных моделей. Общий объем каждой из компьютерных моделей составляет не более 30 Мб.

Созданные нами компьютерные модели однородных линий с потерями и без потерь позволяют: 1) варьировать в широких пределах первичные параметры линии и её длину, действующее значение и частоту напряжения на входе линии; 2) определять характеристические параметры линии (волновое сопротивление $\boldsymbol{Z}_{\boldsymbol{B}}$, коэффициент ослабления $\boldsymbol{\alpha}$, коэффициент фазы $\boldsymbol{\beta}$ ); 3) вычислять действующие напряжения и токи, мощности и входные сопротивления в любой точке линии в режимах холостого хода, короткого замыкания, 
согласованной нагрузки и нагрузочном режиме. Одним из достоинств разработанных нами компьютерных моделей однородной линии является возможность получения графиков распределения действующих и мгновенных значений напряжения и тока вдоль линии.

Необходимость разработки данных компьютерных моделей на языке программирования высокого уровня обусловлена расширением области использования в высшем образовании современных вычислительных технологий в учебном процессе и в научно-исследовательской работе. Создание на основе компьютерных моделей однородной линии виртуальных лабораторных работ позволит более эффективно осваивать учебный материал и использовать полученные теоретические знания на практике.

Основные теоретические положения. Для создания компьютерных моделей применялись основные положения теории цепей с распределёнными параметрами для однородной двухпроводной линии [5].

Компьютерная модель однородной линии с потерями. Однородную линию с потерями можно рассматривать, как идеализированную модель реальной воздушной двухпроводной линии электропередачи, которая характеризуется первичными параметрами: $\mathbf{R}_{0}-$ сопротивление на единицу длины линии (Ом/км), $\mathbf{G}_{0}$ - проводимость утечки на единицу длины линии (CM/км), $\mathbf{L}_{\mathbf{0}}-$ индуктивность на единицу длины линии (Гн/км), $\mathbf{C}_{0}$ - емкость на единицу длины линии (Ф/км). При написании программы «Компьютерная модель длинной линии» для компьютерного моделирования установившихся режимов работы однородной линии с потерями применялись уравнения однородной линии с гиперболическими функциями, нагруженной на сопротивление $\underline{Z}_{H}$ :

$$
\begin{aligned}
& \underline{\mathrm{U}}(\mathrm{x})=\underline{\mathrm{U}}_{2}\left(\operatorname{ch} \gamma(1-\mathrm{x})+\frac{\underline{\mathrm{Z}}_{\mathrm{B}}}{\underline{\mathrm{Z}}_{\mathrm{H}}} \operatorname{sh} \gamma(1-\mathrm{x})\right) ; \\
& \underline{\mathrm{I}}(\mathrm{x})=\frac{\underline{\mathrm{U}}_{2}}{\underline{\mathrm{Z}}_{\mathrm{B}}}\left(\operatorname{sh} \gamma(1-\mathrm{x})+\frac{\underline{\mathrm{Z}}_{\mathrm{B}}}{\underline{\mathrm{Z}}_{\mathrm{H}}} \operatorname{ch} \gamma(1-\mathrm{x})\right),
\end{aligned}
$$

где $\underline{\mathrm{U}}(\mathrm{x})$ - напряжение в текущей точке линии; $\underline{\mathrm{U}}_{2}-$ напряжение в конце линии; $\underline{\mathrm{I}}(\mathrm{x})$ - ток в текущей точке линии; $\gamma=\sqrt{\left(\mathrm{R}_{0}+\mathrm{j} \omega \mathrm{L}_{0}\right)\left(\mathrm{G}_{0}+\mathrm{j} \omega \mathrm{C}_{0}\right)}=\alpha+\mathrm{j} \beta$ - коэффициент распространения, км $^{-1} ; \alpha-$ коэффициент ослабления, Нп/км; $\beta-$ коэффициент фазы, рад/км; $\underline{\mathrm{Z}}_{\mathrm{B}}=\sqrt{\frac{\mathrm{R}_{0}+\mathrm{j} \omega \mathrm{L}_{0}}{\mathrm{G}_{0}+\mathrm{j} \omega \mathrm{C}_{0}}}=\mathrm{Z}_{\mathrm{B}} \mathrm{e}^{\mathrm{j} \theta}-$ волновое сопротивление, Ом; $\underline{Z}_{H}-$ сопротивление нагрузки, Ом; $l$ - длина линии, км; $\mathbf{X}$ - расстояние текущей точки линии от начала линии, км.

Напряжение в конце линии $\underline{\mathrm{U}}_{2}$ определялось через напряжение в начале линии $\underline{\mathrm{U}}_{1}$, равное напряжению источника питания, по формуле:

$$
\underline{\mathrm{U}}_{2}=\frac{\underline{\mathrm{U}}_{1}}{\operatorname{ch} \gamma 1+\frac{\underline{\mathrm{Z}}_{\mathrm{B}}}{\underline{\mathrm{Z}}_{\mathrm{H}}} \operatorname{sh} \gamma \mathrm{l}} .
$$

Из уравнений однородной линии с потерями (1) получают уравнения для режимов холостого хода, короткого замыкания и согласованной нагрузки.

Компьютерная модель однородной линии без потерь. В радиотехнике широко применяются высокочастотные короткие линии, в которых с достаточно большой точностью можно положить равными нулю сопротивление проводов линии $\mathrm{R}_{0}=0$ и проводимость утечки между проводами $\mathrm{G}_{0}=0$. Такие линии называются линиями без потерь. Первичные параметры линии: $\mathrm{L}_{0}$ - индуктивность на единицу длины линии, мкГн/см; $\mathrm{C}_{0}$ - емкость на единицу длины линии, пФ/см. При написании программы «Исследование различных режимов в однородной линии без потерь с помощью компьютерного моделирования» применялись уравнения однородной линии без потерь, нагруженной на сопротивление $\mathrm{R}_{\mathrm{H}}$ :

$$
\begin{aligned}
& \underline{\mathrm{U}}(\mathrm{x})=\underline{\mathrm{U}}_{2}\left(\cos \beta(1-\mathrm{x})+\mathrm{j} \frac{\mathrm{Z}_{\mathrm{B}}}{\mathrm{R}_{\mathrm{H}}} \sin \beta(1-\mathrm{x})\right) ; \\
& \underline{\mathrm{I}}(\mathrm{x})=\frac{\underline{\mathrm{U}}_{2}}{\mathrm{Z}_{\mathrm{B}}}\left(\mathrm{j} \sin \beta(1-\mathrm{x})+\frac{\mathrm{Z}_{\mathrm{B}}}{\mathrm{R}_{\mathrm{H}}} \cos \beta(1-\mathrm{x})\right),
\end{aligned}
$$


где $\gamma=\mathrm{j} \omega \sqrt{\mathrm{L}_{0} \mathrm{C}_{0}}=\mathrm{j} \beta$ - коэффициент распространения, $\mathrm{cm}^{-1} ; \alpha=0$ - коэффициент ослабления; $\beta=\omega \sqrt{\mathrm{L}_{0} \mathrm{C}_{0}}-$ коэффициент фазы, рад/см; $\mathrm{Z}_{\mathrm{B}}=\sqrt{\frac{\mathrm{L}_{0}}{\mathrm{C}_{0}}}-$ волновое сопротивление, Ом; $R_{H}$ - сопротивление нагрузки, Ом; $l$ - длина линии, см; $\mathrm{x}$ - расстояние текущей точки линии от начала линии, см.

Напряжение в конце линии $\underline{U}_{2}$ определялось через напряжение в начале линии $\underline{U}_{1}$, равное напряжению источника питания, по формуле:

$$
\underline{\mathrm{U}}_{2}=\frac{\underline{\mathrm{U}}_{1}}{\cos \beta 1+\mathrm{j} \frac{\mathrm{Z}_{\mathrm{B}}}{\mathrm{R}_{\mathrm{H}}} \sin \beta 1} .
$$

Из уравнений однородной линии без потерь (3) получают уравнения для режимов холостого хода, короткого замыкания и согласованной нагрузки.

Результаты исследования. Компьютерное моделирование установивиихся режимов работы однородной линии $c$ потерями. Компьютерное моделирование установившихся режимов работы однородной линии с потерями осуществляется с помощью программы «Компьютерная модель длинной линии». Для большей наглядности в программе выделяются различные режимы работы однородной линии с потерями: режимы холостой хода и короткого замыкания, согласованной нагрузки и нагрузочный режим.

После запуска программы «Компьютерная модель длинной линии» открывается главное окно программы, содержащее рабочую область в центре окна, верхнюю и нижнюю панели инструментов, на которых расположены измерительные приборы и управляющие кнопки (рис. 1).

На верхней панели инструментов расположены: группа кнопок для выбора режима работы линии: холостой ход, короткое замыкание, согласованная нагрузка, нагрузочный режим; кнопка «Линия» для ввода параметров линии, кнопка для установки параметров источника питания, кнопка для установки значения сопротивления нагрузки; кнопка, которая открывает окно графиков распределения вдоль однородной линии действующих значений напряжения и тока «Графики $\mathrm{U}(\mathrm{x})$, I(x)». На верхней панели также расположены: 1) окно, в котором отображаются характеристические параметры линии: модуль $Z_{B}$ и аргумент $\theta$ волнового сопротивления, коэффициент затухания и коэффициент фазы; 2) окно, в котором отображаются модуль Z(x) и аргумент $\varphi(\mathrm{x})=\psi_{\mathrm{U}}(\mathrm{x})-\psi_{\mathrm{I}}(\mathrm{x})$ входного сопротивления, начальные фазы напряжения $\psi_{\mathrm{U}}(\mathrm{x})$ и тока $\psi_{\mathrm{I}}(\mathrm{x})$.

Для выбора режима работы линии выделяем требуемый режим и щелкаем левой кнопкой мыши. В рабочей области главного окна появляется схема двухпроводной линии в заданном режиме (рис. 1).

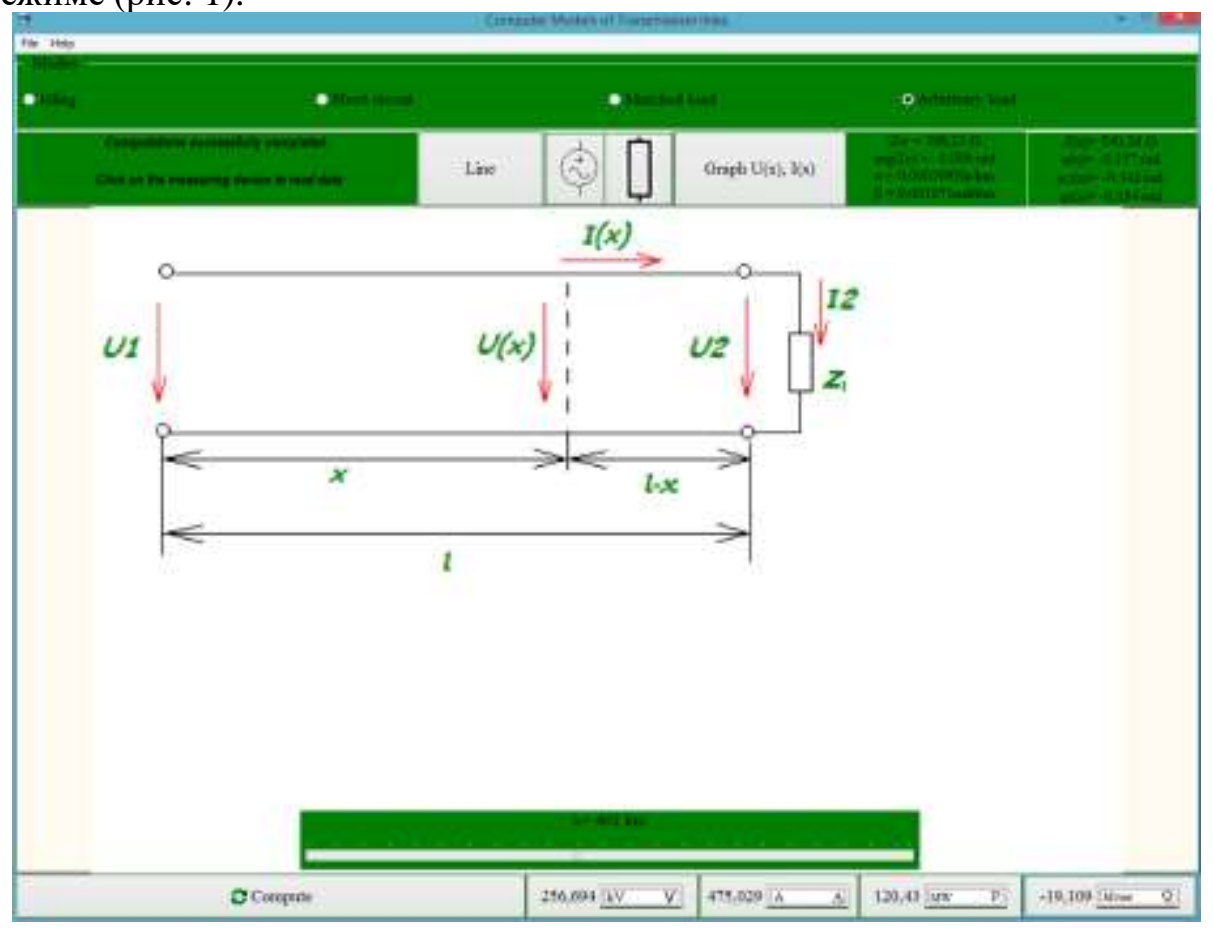

Рис. 1. Главное окно программы в нагрузочном режиме. 
При нажатии на кнопку «Линия», открывается окно, в котором вводят значения длины линии и ее первичные параметры. Чтобы открыть окно для ввода величины и частоты напряжения источника питания, нажимают на кнопку, изображающую источник. Для ввода модуля и аргумента сопротивления нагрузки щелкают по кнопку с изодражением сопротивления. На рисунке 2 показаны открытые окна «Первичные параметры и длина линии», «Напряжение и частота источника», «Нагрузка».

На нижней панели инструментов расположены кнопка «Вычислить» для включния измерительных приборов и кнопки измерительных приборов: вольтмера «V», амперметра «А», ваттметра «Р» и прибора для измерения реактивной мощности - варметра «Q». Так как программа «Компьютерная модель длинной линии» предназначена для моделирования высоковольтных ЛЭП, то мы установили единицы измерения напряжения кВ, активной мощности МВт, реактивной МВАр.

Внизу рабочей области под изображением линии расположен бугунок, с помощью которого можно устанавливать текущие точки линии. Для того чтобы измерить напряжение, ток, активную и реактивную мощности, необходимо установить бегунок в заданную текущую точку линии с координатой х, нажать кнопку на нижней панели инструментов «Вычислить», а также на кнопки «V», «A», «Р», «Q». Рядом с измерительными приборами появляются действующие значения напряжения и тока, а также значения активной и реактивной мощностей в выбранной точке однородной линии (рис. 1, 2). Результаты вычислений можно экспортировать в Word -файл.

Перемещая бегунок вдоль линии и измеряя в каждой точке линии действующие значения напряжения $\mathrm{U}(\mathrm{x})$ и тока $I(x)$, а также активную $P(x)$ и реактивную $\mathrm{Q}(\mathrm{x})$ мощности, модуль $Z(x)$ и аргумент $\varphi(x)=\psi_{U}(\mathrm{x})-\psi_{I}(\mathrm{x})$ входного сопротивления можно получить их распределение вдоль всей линии.

Для получения графиков действующих значений напряжения $\mathrm{U}(\mathrm{x})$, и тока $\mathrm{I}(\mathrm{x})$, нажимают на кнопку «Графики $\mathrm{U}(\mathrm{x}), \mathrm{I}(\mathrm{x}) »$. Графики можно перемещать с помощью правой кнопки мыши. Графики сохраняются в формате bmp-файла.

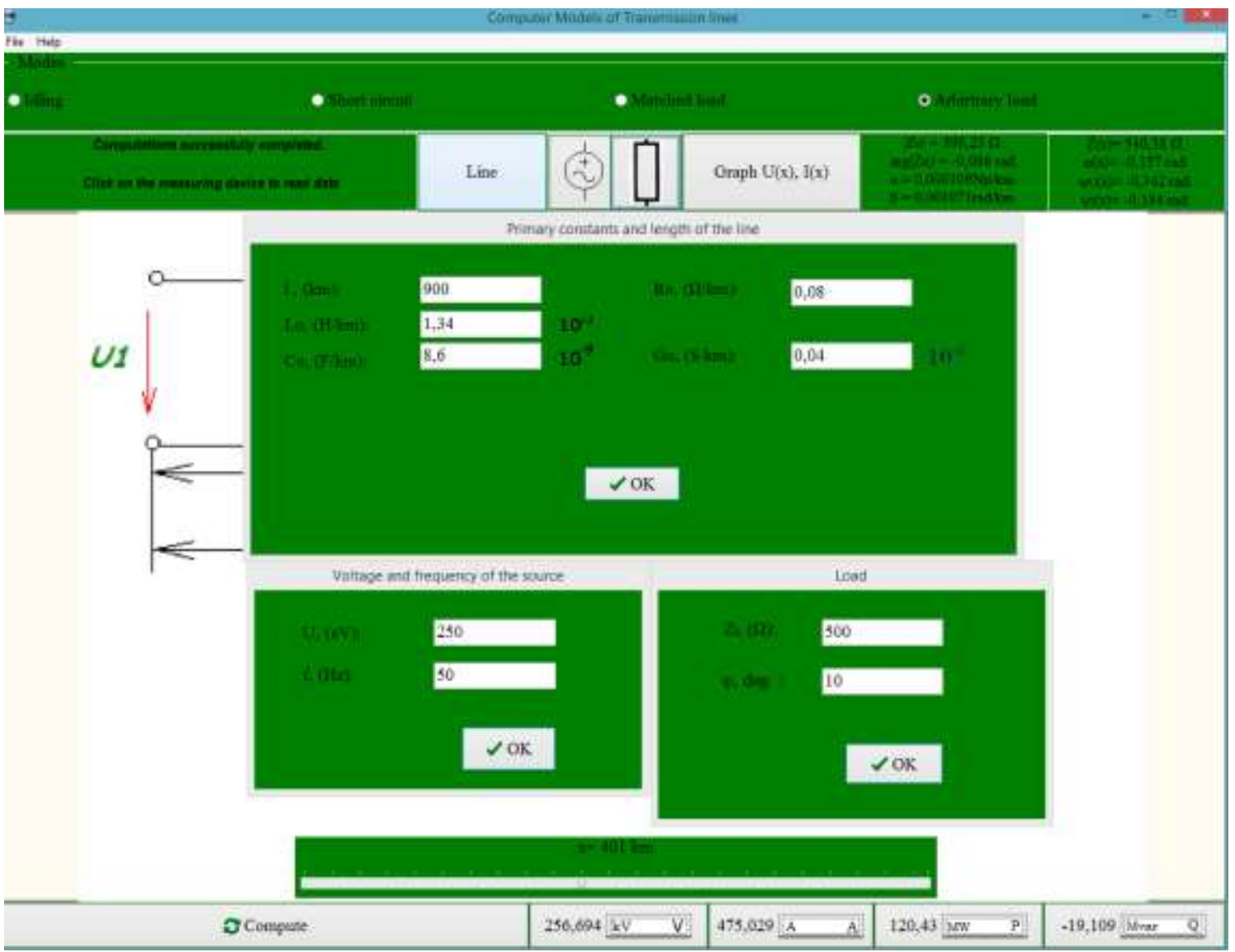

Рис. 2. Главное окно программы в нагрузочном режиме с открытыли окнами: «Первичные параметры и длина линии», «Напряжение и частота источника», «Нагрузка». 


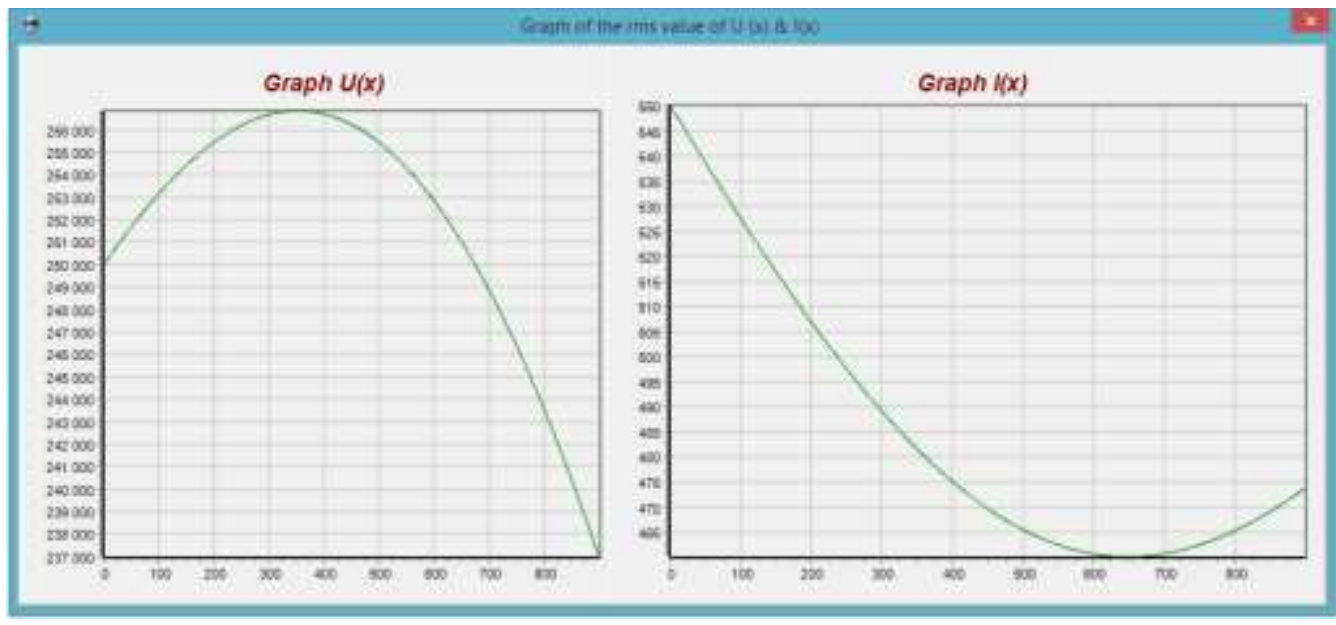

Рис. 3 График распределения действующих значений напряжения и тока вдоль линии в нагрузочном режиме

На рисунке 3 приведены графики распределения действующих значений напряжения и тока вдоль линии длиной 900 км в нагрузочном режиме, когда сопротивление нагрузки не равно волновому сопротивлению.

По вычисленным значениям активной $\mathrm{P}(\mathrm{x})$ и реактивной $\mathrm{Q}(\mathrm{x})$ мощностей, модуля $\mathrm{Z}(\mathrm{x})$ и аргумента $\varphi(\mathrm{x})$ входного сопротивления линии можно построить графики распределения $\mathrm{P}(\mathrm{x}), \mathrm{Q}(\mathrm{x}), \mathrm{Z}(\mathrm{x})$ и $\varphi(\mathrm{x})$ вдоль линии. На рисунке 4 приведены графики распределения активной и реактивной мощностей вдоль линии в нагрузочном режиме.

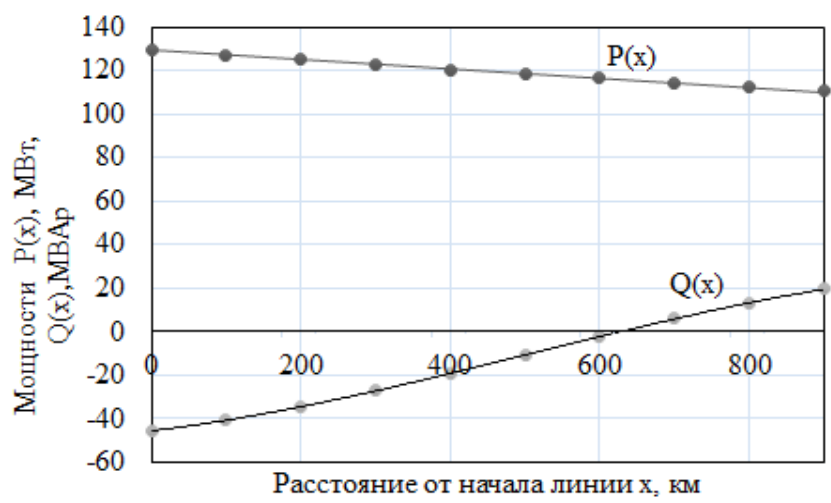

Рис. 4. График распределения активной и реактивной мощностей вдоль линии в нагрузочном режсиме

Аналогично нагрузочному режиму исследуются установившиеся режимы холостого хода, короткого замыкания и согласованной нагрузки.

Изменяя параметры однородной линии, сопротивление нагрузки можно исследовать изменения напряжения, тока, мощностей, входного сопротивления линии и предложить рекомендации по оптимизации её работы.

Компьютерное моделирование установившихся режсимов работы однородной линии без nотерь. Компьютерное моделирование установившихся режимов работы однородной линии без потерь осуществляется с помощью программы «Исследование различных режимов в однородной линии без потерь с помощью компьютерного моделирования». Для большей наглядности в программе выделяются различные режимы работы однородной линии без потерь: режимы холостой хода и короткого замыкания, согласованной нагрузки и нагрузочный режим.

После запуска программы «Исследование различных режимов в однородной линии без потерь» открывается главное окно программы, содержащее рабочую область в центре окна и две боковые панели инструментов. На боковых панелях инструментов расположены измерительные приборы и управляющие кнопки (рисунок 5). На левой боковой панели инструментов расположена группа кнопок для выбора режима работы однородной линии без потерь: режимы холостого хода, короткого замыкания, согласованной нагрузки и нагрузочный режим. Для выбора режима работы линии выделяем требуемый режим и щелкаем левой 
кнопкой мыши. В рабочей области главного окна появляется схема двухпроводной линии в заданном режиме (на рисунке 5 выбран режим холостого хода).

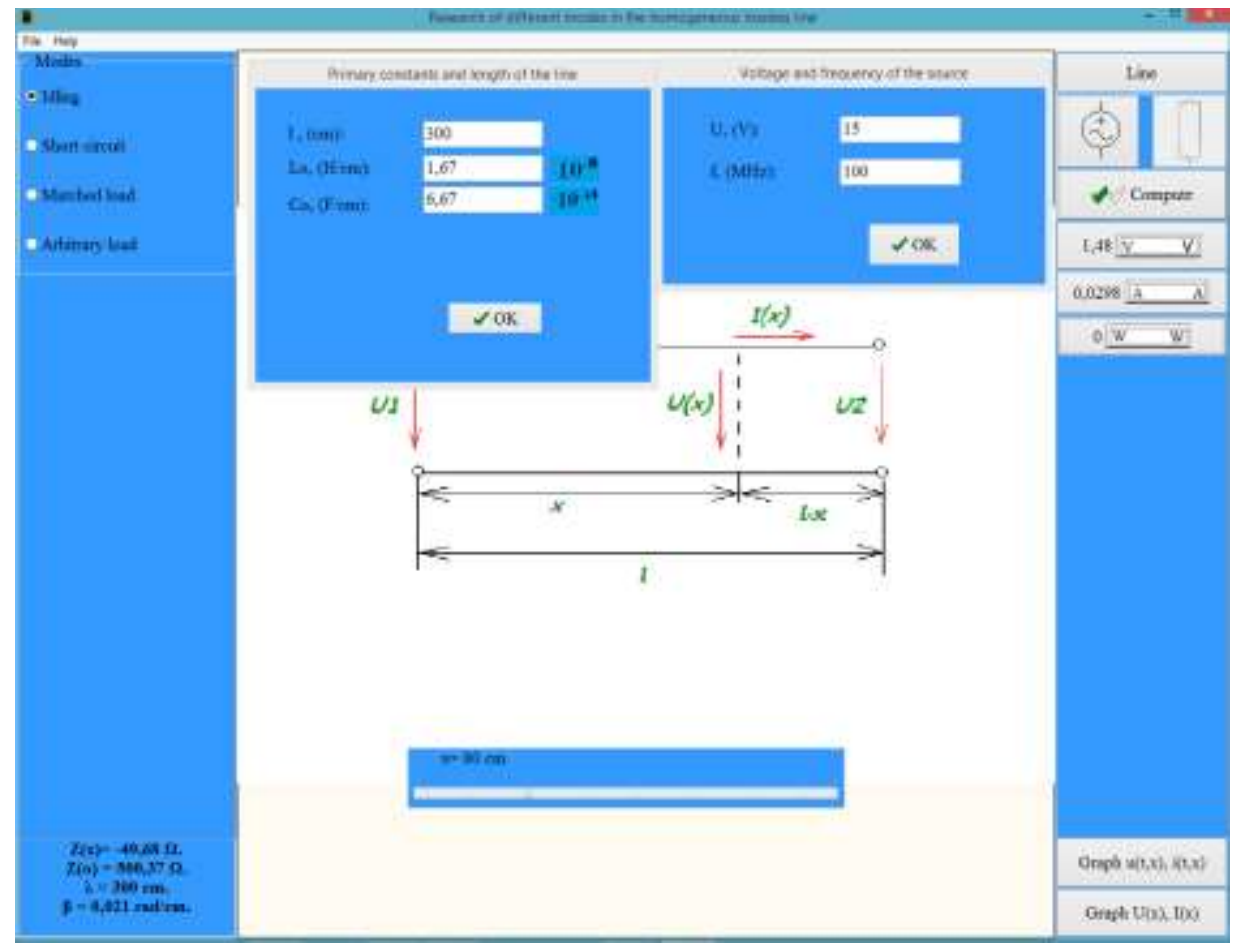

Рис. 5. Главное окно программы в режиме холостого хода с открытыми окнами: «Первичные параметры и длина линии», «Напряжсение и частота источника».

На правой боковой панели расположены: кнопка «Линия» для ввода параметров линии; кнопка с изображением источника для установки параметров источника питания; кнопка с изображением сопротивления для установки значения сопротивления нагрузки; кнопка «Расчет» для определения действующих значений напряжения, тока и активной мощности в текущей точке однородной линии без потерь. При нажатии на кнопку «Линия», открывается окно, в котором вводят значения длины линии без потерь и ее первичные параметры. Чтобы открыть окно для ввода величины и частоты напряжения источника питания, нажимают на кнопку, изображающую источник. Для ввода активного сопротивления нагрузки $\mathrm{R}_{\mathrm{H}}$ щелкают по кнопку с изодражением сопротивления. На рисунке 5 показаны открытые окна «Первичные параметры и длина линии», «Напряжение и частота источника».

На правой панели инструментов расположены кнопка «Расчет» для включния измерительных приборов и кнопки измерительных приборов: вольтмера «V», амперметра «А», ваттметра «W».

В рабочей области под изображением линии расположен бугунок, с помощью которого можно устанавливать текущие точки линии. Для того чтобы измерить напряжение, ток, активную мощность необходимо установить бегунок в заданную текущую точку линии с координатой х, нажать кнопку на правой панели инструментов «Расчет», а также на кнопки «V», «A», «W» (рисунок 5). Рядом с приборами появляются вычисленные действующие значения напряжения, тока и активной мощности. Результаты вычислений можно экспортировать в Word-файл.

Внизу левой боковой панели отображаются также значения входное сопротивление линии в текущей точке, волновое сопротивление, коэффициент фазы и длина волны электромагнитных колебаний.

Перемещая бегунок вдоль линии и измеряя в каждой точке линии действующие значения напряжения $\mathrm{U}(\mathrm{x})$ и тока $I(x)$, а также активную $P(x)$ мощность, входное сопротивление $Z(x)$ можно получить распределение этих величин вдоль всей линии.

Внизу на правой панели расположены: кнопка «Графики $\mathrm{U}(\mathrm{x}), \mathrm{I}(\mathrm{x}) »$, открывающая окно графиков распределения действующих значений напряжения и тока вдоль линии; кнопка, «Графики $\mathrm{u}(\mathrm{t}, \mathrm{x}), \mathrm{i}(\mathrm{t}, \mathrm{x}) »$ открывающая окно графиков распределения мгновенных напряжений и токов вдоль длины линии в различные моменты времени.

Чтобы получить графики действующих значений напряжения и тока, нажимаем на кнопку «Графики $\mathrm{U}(\mathrm{x}), \mathrm{I}(\mathrm{x}) »$. В открывшемся окне отображаются графики действующих 
значений $\mathrm{U}(\mathrm{x})$, I(x). На рисунке 6 приведены графики действующих значений напряжения и тока в режиме холостого хода.

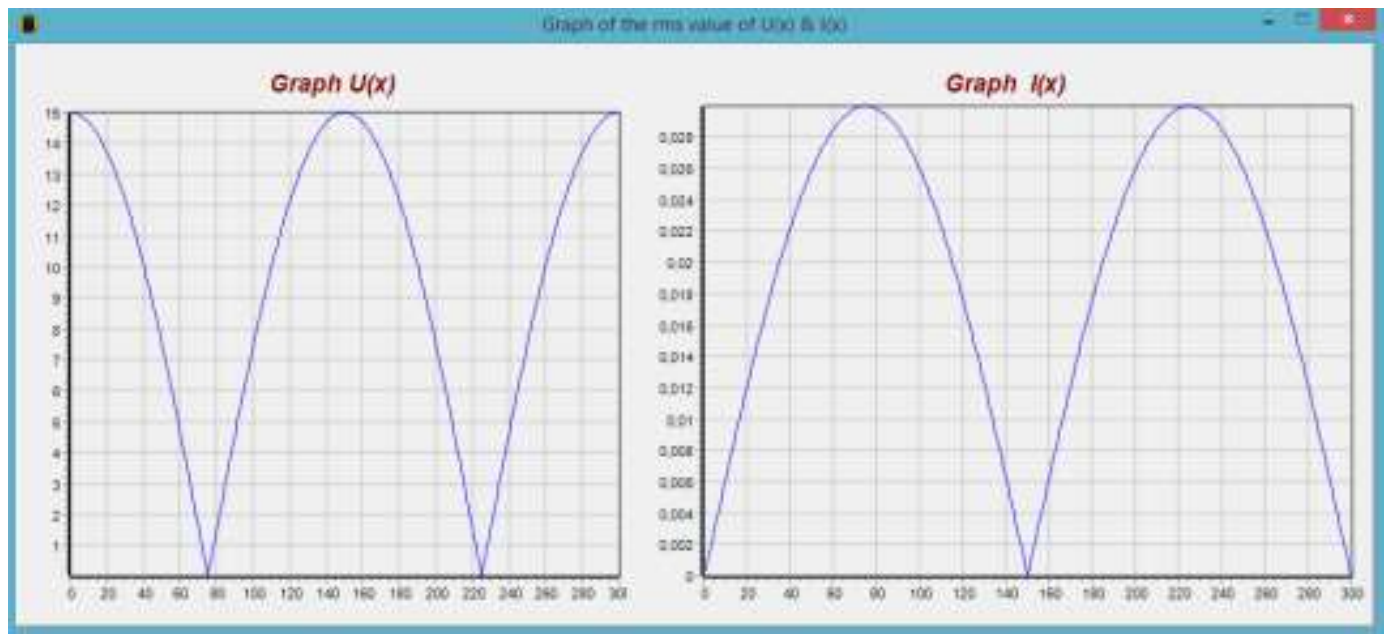

Рис. 6. Графики действующих значений напряжения и тока в режиме холостого хода.

Для построения графиков распределения вдоль длины линии мгновенных значений напряжения $\mathrm{u}(\mathrm{t}, \mathrm{x})$ и тока $\mathrm{i}(\mathrm{t}, \mathrm{x})$ в различные моменты времени нажимаем левой кнопкой мыши на кнопку «Графики $\mathrm{u}(\mathrm{t}, \mathrm{x}), \mathrm{i}(\mathrm{t}, \mathrm{x}) »$. В полученном окне щелкаем по кнопке «Построить» и затем поочередно по кнопкам, соответствующим различным моментам времени: 0, T/8, T/4, T/2, 3Т/8, 3T/4. На рисунке 7 приведены графики распределения вдоль длины линии мгновенных значений напряжения $\mathrm{u}(\mathrm{t}, \mathrm{x})$ и тока $\mathrm{i}(\mathrm{t}, \mathrm{x})$ в различные моменты времени в режиме холостого хода.

Графики действующих и мгновенных значений напряжения и тока в режиме холостого хода наглядно иллюстрируют режим стоячих волн в линии без потерь. Как видно из приведенных графиков узлы и пучности напряжения и тока неподвижны, причем пучности напряжения совпадают с узлами тока.

Аналогичные графики распределения вдоль линии напряжения и тока получаются в режиме стоячих волн при коротком замыкании линии без потерь.

Если выбрать режим согласованной нагрузки и построить графики распределения мгновенных значений напряжения и тока в различные моменты времени, то видно, что кривые распределения мгновенных значений напряжения и тока вдоль линии являются синусоидальными и перемещаются по линии от начала линии к её концу. Графики мгновенных значений в режиме согласованной нагрузки наглядно иллюстрируют прямые бегущие волны напряжения и тока (рис. 8).

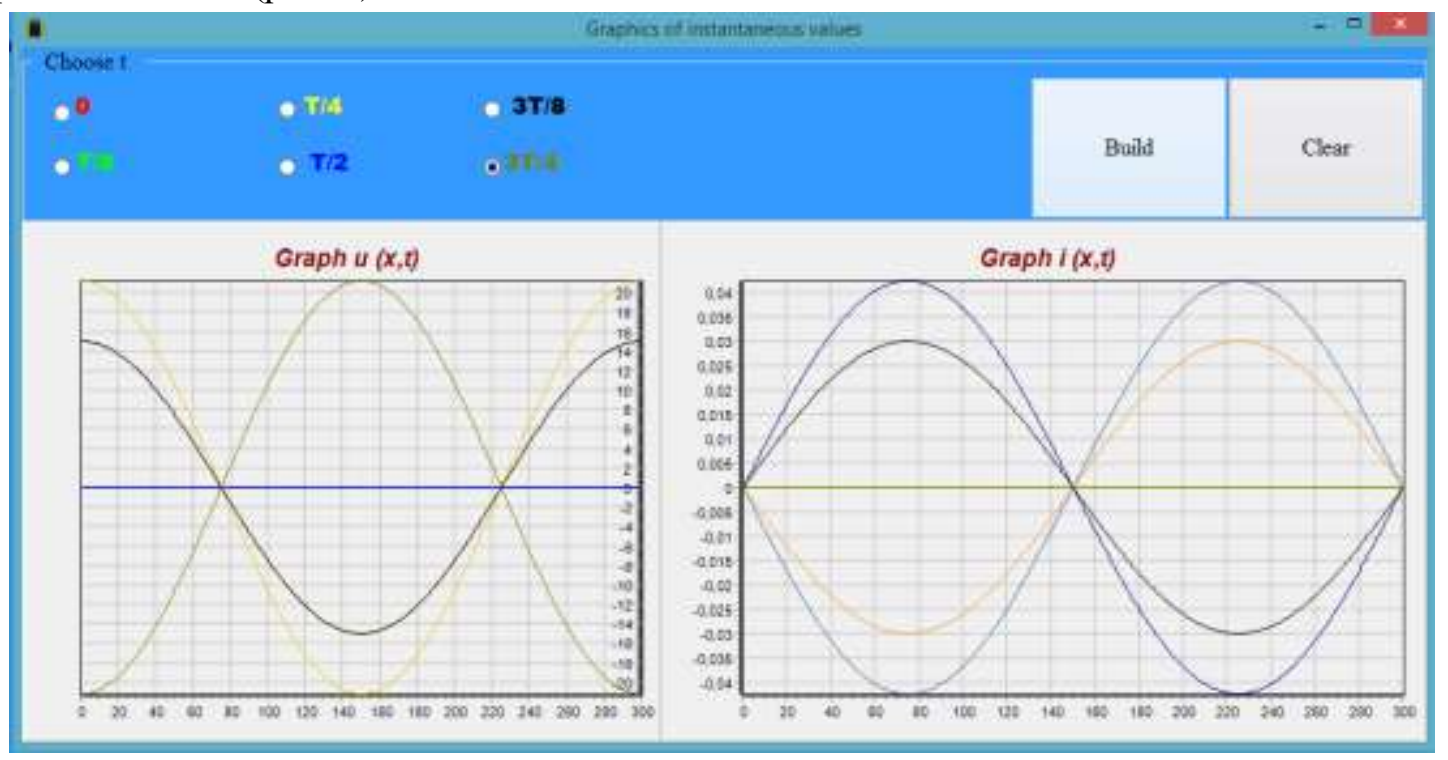

Рис. 7. Графики распределения вдоль линии мгновенных значений напряжения и тока в режиме холостого хода 


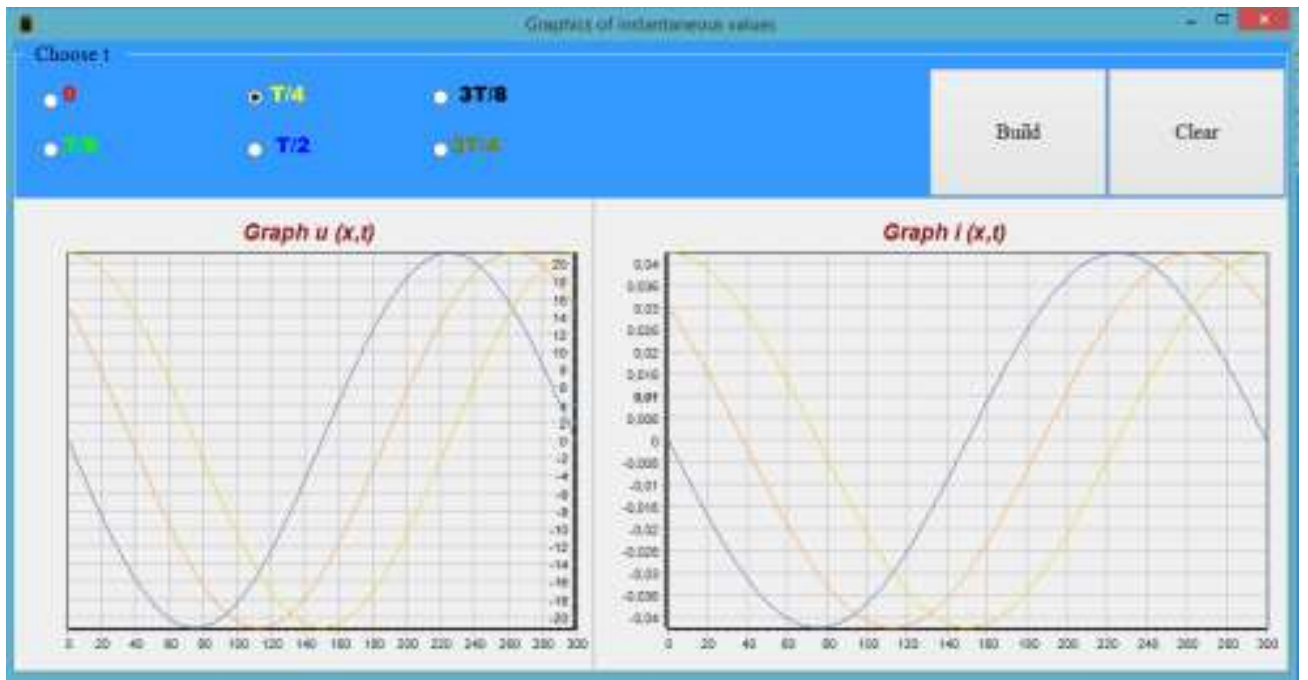

Рис. 8. Графики распределения вдоль линии мгновенных значений напряжения и тока в режиме согласованной нагрузки

Графики распределения вдоль линии мгновенных значений напряжения и тока удаляются с помощью кнопки «Очистить» и их можно перемещать с помощью правой кнопки мыши.

Графики распределения вдоль линии действующих и мгновенных значений напряжения и тока сохраняются в формате bmp-файла.

Для исследования нагрузочного режима щелкают по кнопке «Нагрузочный режим» и задают величину сопротивления нагрузки, используя кнопку с изображением схемы сопротивления.

Выводы. Созданы программы: «Компьютерная модель длинной линии», которая применяется для моделирования установившихся режимов работы однородный линии с потерями и «Исследование различных режимов в однородной линии без потерь с помощью компьютерного моделирования», которая позволяет всесторонне исследовать однородную линию без потерь.

Программное обеспечение разработана на основе компилируемого языка программирования высокого уровня Delphi в среде Embarcadero RAD Studio 2010. Созданные компьютерные программы имеют оригинальные, дружественные интерфейсы, отличаются простотой в использовании, быстры и надежны в работе, является «неприхотливым» и устойчивым инструментом для расчета основных параметров однородной линии с потерями и без потерь, с большими возможностями работы. Также обладает в полном объеме средствами, позволяющими обеспечить максимальное выполнение операций, реализованных в компьютерных моделях.

Программные продукты «Компьютерная модель длинной линии» и «Исследование различных режимов в однородной линии без потерь с помощью компьютерного моделирования〉 могут быть использованы в научно-исследовательской работе и в учебном процессе для создания на их основе виртуальных лабораторных работ по компьютерному моделированию установившихся режимов работы однородной линии.

\section{ЛИТЕРАТУРА}

1. Карлащук В.И. Электронная лаборатория на IBM РС. Программа Electronics Workbench и её применение. М.: Солон-Пресс, 1999. 506 с.

2. Денисенко В. И., Зуслина Е.Х. Кондратенко Л. Н. Компьютерное моделирование цепей с распределенными параметрами в лабораторном практикуме по теории электрических цепей // Tp. Междунар. научно-технической конф. «Энергетика, телекоммуникации и высшее образование в современных условиях». Алматы: АИЭС, 2004. с. 132-134.

3. Зуслина Е.Х., Утебалиева Б. Д. Компьютерная модель длинной линии. Свидетельство о государственной регистрации прав на объект авторского права ИС № 0010428. Астана, Комитет по правам интеллектуальной собственности МЮ РК, 2013.

4. Зуслина Е.Х., Утебалиева Б. Д. Исследование различных режимов в однородной линии без потерь с помощью компьютерного моделирования. Свидетельство о государственной регистрации прав на объект авторского права ИС № 0009832. Астана, Комитет по правам интеллектуальной собственности МЮ РК, 2013.

5. Основы теории цепей. Учебник для вузов / Г.В Зевеке, П.А. Ионкин А.В. Нетушил, С.В. Страхов. М.: Энергоатомиздат, 1989. 528 с.

6. Фленов М.Е. Библия Delphi. Петербург: БХВ, 2011. 688 с.

7. Санников Е. В. Курс практического программирования в Delphi. Объектно-ориентированное программирование. Петербург: Солон-Пресс, 2013. 188 с. 